\title{
Neutral functional sequential differential equations with Caputo fractional derivative on time scales
}

\author{
Jamal Eddine Lazreg' ${ }^{1}$ Nadia Benkhettou', Mouffak Benchohra' and Erdal Karapinar ${ }^{2,3^{*}}$ (D)
}

\section{"Correspondence:}

erdalkarapinar@tdmu.edu.vn erdalkarapinar@yahoo.com

${ }^{2}$ Division of Applied Mathematics,

Thu Dau Mot University, Thu Dau

Mot, Binh Duong Province, Vietnam

${ }^{3}$ Department of Mathematics,

Çankaya University, 06790,

Etimesgut, Ankara, Turkey

Full list of author information is

available at the end of the article

\section{Springer}

\begin{abstract}
In this paper, we establish the existence and uniqueness of a solution for a class of initial value problems for implicit fractional differential equations with Caputo fractional derivative. The arguments are based upon the Banach contraction principle, the nonlinear alternative of Leray-Schauder type and Krasnoselskii fixed point theorem. As applications, two examples are included to show the applicability of our results.
\end{abstract}

MSC: $26 \mathrm{~A} 33 ; 34 \mathrm{~A} 08$

Keywords: Initial value problem; Caputo's fractional derivative; Sequential neutral functional differential equations; Fractional integral; Fixed point; Existence; Time scale

\section{Introduction}

For the past decades, fractional differential equations have been of increasing importance due to their diverse applications in different fields, such as control theory, electrochemistry, viscoelasticity, electromagnetism, biology, economics, quantum calculus, etc. (see $[2-10,15,17,29-31,34]$ and the references therein). On the other hand, the theory of differential equations on time scales has developed very intensively during the last decades (see for example [11, 12, 18-22, 24-27, 33] and the references therein). In 1988, Stefan Hilger $[16,28]$ introduced in his thesis the concept of "calculation of chains of measures" in order to unify the discrete and continuous analysis. So, all results found will be valid in the discrete case and in the continuous case.

In [32], using suitable fixed point theorems, Vipin and Muslim established the existence and uniqueness of the solutions to the following nonlinear fractional differential equation with nonlinear integral boundary conditions on time scales:

$$
\begin{aligned}
& { }^{c} \Delta^{\vartheta} u(\theta)=\Psi(\theta, u(\alpha(\theta))), \quad \theta \in \mathcal{J}=[0, T]_{\mathbb{T}}, \vartheta \in(0,1), \\
& \beta u(0)+\eta u(T)=\frac{1}{\Gamma(\vartheta)} \int_{0}^{T}(T-s)^{\vartheta-1} g(s, u(s)) \Delta s,
\end{aligned}
$$

(c) The Author(s) 2022. This article is licensed under a Creative Commons Attribution 4.0 International License, which permits use, sharing, adaptation, distribution and reproduction in any medium or format, as long as you give appropriate credit to the original author(s) and the source, provide a link to the Creative Commons licence, and indicate if changes were made. The images or other third party material in this article are included in the article's Creative Commons licence, unless indicated otherwise in a credit line to the material. If material is not included in the article's Creative Commons licence and your intended use is not permitted by statutory regulation or exceeds the permitted use, you will need to obtain permission directly from the copyright holder. To view a copy of this licence, visit http://creativecommons.org/licenses/by/4.0/ 
where ${ }^{c} \Delta^{\vartheta}$ is the Caputo delta-fractional derivative and $0, T \in \mathbb{T}, \beta, \eta \in \mathbb{R}$ such that $\beta+\eta \neq$ $0, \alpha: \mathcal{J} \rightarrow \mathcal{J}$ is a continuous function with $\alpha(\theta) \leq \theta, \Psi$ and $g$ are some functions. In [32], the authors discussed the existence, uniqueness, and stability for the nonlinear fractional differential equations with impulses on time scales:

$$
\begin{aligned}
& { }^{c} \Delta^{q} \varphi(\eta)=\mathcal{G}\left(\eta, \varphi(\eta),{ }^{c} \Delta^{q} \varphi(\eta)\right), \quad \eta \in \mathcal{J}=[0, T]_{\mathrm{T}}, \eta \neq \eta_{l}, \\
& \varphi\left(\eta_{l}^{+}\right)-\varphi\left(\eta_{l}^{-}\right)=I_{l}\left(\eta_{l}, \varphi\left(\eta_{l}^{-}\right)\right), \quad l=1,2, \ldots, p, \\
& \varphi(0)=\varphi_{0},
\end{aligned}
$$

where $\mathbb{T}$ is a time scale with $0, \eta_{l} \in \mathbb{T},{ }^{c} \Delta^{q}$ is the Caputo fractional derivative with $q \in(0,1)$ and $l=1,2, \ldots, p$, the point of impulses $\eta_{k}$ is right dense with $0 \leq \eta_{0}<\eta_{1}<\eta_{2}<\cdots<\theta_{p}<$ $\eta_{p+1}=T, \varphi\left(\eta_{k}^{-}\right)=\lim _{h \rightarrow 0^{+}} \varphi\left(\eta_{k}-h\right), \varphi\left(\eta_{k}^{+}\right)=\lim _{h \rightarrow 0_{+}} \varphi\left(\eta_{k}+h\right)$ denote the left and the right limits of $\varphi(\eta)$ at $\eta=\eta_{k}, I_{k} \in C(\mathcal{J} \times \mathbb{R}, \mathbb{R})$ and $\mathcal{G}: \mathcal{J} \times \mathbb{R} \times \mathbb{R} \rightarrow \mathbb{R}$ are given functions. In [23], Vivek et al. proved some existence and stability results of Hilfer-type fractional differential equations on time scales

$$
\left\{\begin{array}{l}
{ }_{\mathbb{T}} \Delta_{0^{+}}^{\alpha, \beta} \eta(t)=\mathcal{F}(t, \eta(t)), \quad t=[0, T]:=\mathcal{J} \subseteq \mathbb{T}, T>0, \\
{ }^{\mathbb{T}} I_{0^{+}}^{1-\gamma} \eta(0)=\eta_{0}, \quad \gamma=\alpha+\beta-\alpha \beta,
\end{array}\right.
$$

where ${ }^{\mathbb{T}} \Delta_{0^{+}}^{\alpha, \beta}$ is the Hilfer fractional differential defined on a time scale $\mathbb{T}, 0<\alpha<1,0 \leq$ $\beta \leq 1$ and $\mathcal{F}: \mathcal{J} \times \mathbb{T} \rightarrow \mathbb{R}$ is a right-dense continuous function.

In [13], Ahmad and Ntouyas proved some existence results for the following initial value problem of Caputo-Hadamard sequential fractional order neutral functional differential equations:

$$
\left\{\begin{array}{l}
\mathfrak{D}^{p}\left[\mathfrak{D}^{q} \varpi(\vartheta)-G\left(\vartheta, \varpi_{\vartheta}\right)\right]=F\left(\vartheta, \varpi_{\vartheta}\right), \quad \vartheta \in \mathbf{J}:=[1, T], \\
\varpi(\vartheta)=\phi(\vartheta), \quad \vartheta \in[1-\sigma, 1], \\
\mathfrak{D}^{q} \varpi(1)=\eta \in \mathbb{R},
\end{array}\right.
$$

where $\mathfrak{D}^{p}, \mathfrak{D}^{q}$ are the Caputo-Hadamard fractional derivatives, $0<p, q<1, F, G: \mathbf{J} \times$ $C([-\sigma, 0], \mathbb{R}) \rightarrow \mathbb{R}$ are given functions, and $\phi \in C([1-\sigma, 1], \mathbb{R})$, where $\sigma>0$.

In this paper, we generalize the problem considered in [13] to time scales, and we discuss existence and uniqueness of solutions to the following Cauchy problem of Caputo sequential fractional order neutral functional differential equations on time scale $\mathbb{T}$ :

$$
\begin{aligned}
& { }^{c} \Delta^{\omega}\left[{ }^{c} \Delta^{\varpi} u(\vartheta)-\Phi\left(\vartheta, u_{\vartheta}\right)\right]=\Psi\left(\vartheta, u_{\vartheta}\right), \quad \vartheta \in \mathfrak{J}:=[0, T]_{\mathbb{T}}=[0, T] \cap \mathbb{T}, \\
& u(\vartheta)=\zeta(\vartheta), \quad \vartheta \in[-\epsilon, 0]_{\mathbb{T}}=[-\epsilon, 0] \cap \mathbb{T}, \\
& { }^{c} \Delta^{\varpi} u(0)=\phi \in \mathbb{R},
\end{aligned}
$$

where ${ }^{c} \Delta^{\omega},{ }^{c} \Delta^{\varpi}$ are the Caputo fractional derivatives, $0<\omega, \varpi<1, \Psi, \Phi: \mathfrak{J} \times C\left([-\epsilon, 0]_{\mathbb{T}}\right.$, $\mathbb{R}) \rightarrow \mathbb{R}$ are given functions, and $\zeta \in C\left([-\epsilon, 0]_{\mathbb{T}}, \mathbb{R}\right)$. For any function $u$ defined on $[-\epsilon, T]_{\mathbb{T}}$ and any $\vartheta \in \mathfrak{J}$ and $\epsilon>0$, we denote by $u_{\vartheta}$ the element of $C_{\epsilon}:=C\left([-\epsilon, 0]_{\mathbb{T}}, \mathbb{R}\right)$ defined by

$$
u_{\vartheta}(\theta)=u(\vartheta+\theta), \quad \theta \in[-\epsilon, 0]_{\mathbb{T}} .
$$


The present paper is organized as follows. In Sect. 2, some notations are introduced. In Sect. 3, three results for problem (1)-(3) are proved by using the following fixed point theorems: the Banach contraction principle, the nonlinear alternative of Leray-Schauder type, and Krasnoselskii's fixed point theorem. Finally, in the last section, we give two examples to illustrate the applicability of our results.

\section{Preliminaries}

In this section, we collect notations, definitions, and results which are needed in the sequel.

Let $C(\mathfrak{J}, \mathbb{R})$ be the Banach space of all continuous functions from $\mathfrak{J}$ into $\mathbb{R}$ with the norm

$$
\|y\|_{\infty}:=\sup \{|y(\vartheta)|: \vartheta \in \mathfrak{J}\}
$$

Also $C_{\epsilon}$ is endowed with the norm

$$
\|\zeta\|_{C}:=\sup \{|\zeta(\iota)|:-\epsilon \leq \iota \leq 0\}
$$

\subsection{Some properties of time scale}

A time scale $\mathbb{T}$ is an arbitrary nonempty closed subset of $\mathbb{R}$ (for more details, see [2022, 24-27]).

Definition 2.1 Let $\mathbb{T}$ be a time scale. For $\tau \in \mathbb{T}$, we define the forward jump operator $\sigma: \mathbb{T} \rightarrow \mathbb{T}$ by $\sigma(\tau):=\inf \{s \in \mathbb{T}: s>\tau\}$, and the backward jump operator $\rho: \mathbb{T} \rightarrow \mathbb{T}$ by $\rho(\tau):=\sup \{s \in \mathbb{T}: s<\tau\}$.

Remark 2.2 In Definition 2.1, we put $\inf \emptyset=\sup \mathbb{T}$ (i.e., $\sigma(\bar{m})=\bar{m}$ if $\mathbb{T}$ has a maximum $\bar{m}$ ) and $\sup \emptyset=\inf \mathbb{T}$ (i.e., $\rho(\underline{m})=\underline{m}$ if $\mathbb{T}$ has a minimum $\underline{m}$ ), where $\emptyset$ denotes the empty set.

If $\sigma(\tau)>\tau$, then we say that $\tau$ is right-scattered; if $\rho(\tau)<\tau$, then $\tau$ is said to be leftscattered. Points that are simultaneously right-scattered and left-scattered are called isolated. If $\tau<\sup \mathbb{T}$ and $\sigma(\tau)=\tau$, then $\tau$ is called right-dense; if $\tau>\inf \mathbb{T}$ and $\rho(\tau)=\tau$, then $\tau$ is called left-dense. The derivative makes use of the set $\mathbb{T}^{\kappa}$, which is derived from the time scale $\mathbb{T}$ as follows: if $\mathbb{T}$ has a left-scattered maximum $\bar{m}$, then $\mathbb{T}^{\kappa}:=\mathbb{T} \backslash\{\bar{m}\}$; otherwise, $\mathbb{T}^{\kappa}:=\mathbb{T}$.

Definition $2.3([21,22])$ Assume $h: \mathbb{T} \rightarrow \mathbb{R}$ and let $\tau \in \mathbb{T}^{\kappa}$. We define

$$
h^{\Delta}(\tau):=\lim _{s \rightarrow \tau} \frac{h(\sigma(s))-h(\tau)}{\sigma(s)-\tau}, \quad \tau \neq \sigma(s)
$$

provided the limit exists. We call $h^{\Delta}(\tau)$ the delta derivative (or Hilger derivative) of $h$ at $\tau$. Moreover, we say that $h$ is delta differentiable on $\mathbb{T}^{\kappa}$ provided $h^{\Delta}(\tau)$ exists for all $\tau \in \mathbb{T}^{\kappa}$. The function $h^{\Delta}: \mathbb{T}^{\kappa} \rightarrow \mathbb{R}$ is then called the (delta) derivative of $h$ on $\mathbb{T}^{\kappa}$.

Definition 2.4 A function $h: \mathbb{T} \rightarrow \mathbb{R}$ is called rd-continuous provided it is continuous at right-dense points in $\mathbb{T}$ and its left-sided limits exist (finite) at left-dense points in $\mathbb{T}$. $\mathcal{C}_{r d}$ denotes the set of rd-continuous functions $h: \mathbb{T} \rightarrow \mathbb{R}$. 
Definition 2.5 Let $[a, b]$ denote a closed bounded interval in $\mathbb{T}$. A function $H:[a, b] \rightarrow \mathbb{R}$ is called a delta antiderivative of function $h:[a, b) \rightarrow \mathbb{R}$ provided $H$ is continuous on $[a, b]$, delta differentiable on $[a, b)$, and $H^{\Delta}(\tau)=f(\tau)$ for all $\tau \in[a, b)$. Then we define the $\Delta$ integral of $h$ from $a$ to $b$ by

$$
\int_{a}^{b} h(\tau) \Delta \tau:=H(b)-H(a)
$$

Lemma 2.6 ([14]) Suppose that $\mathbb{T}$ is a time scale and $h$ is an increasing continuous function on the time-scale interval $[a, b]$. If $H$ is the extension of $h$ to the real interval $[a, b]$ given by

$$
H(s):= \begin{cases}h(s) & \text { if } s \in \mathbb{T}, \\ h(\tau) & \text { if } s \in(t, \sigma(\tau)) \notin \mathbb{T},\end{cases}
$$

then

$$
\int_{a}^{b} h(\tau) \Delta \tau \leq \int_{a}^{b} H(\tau) d \tau
$$

\subsection{Some properties of fractional calculus on time scales}

We introduce a new notion of fractional derivative on time scales.

Definition 2.7 (Fractional integral on time scales) Suppose that $\mathbb{T}$ is a time scale, $[a, b] \subset$ $\mathbb{T}$, and $\zeta$ is an integrable function on $[a, b]$. Let $0<\omega<1$. Then the fractional integral of order $\omega$ of $\zeta$ is defined by

$$
{ }^{\mathbb{T}} I_{a}^{\omega} \zeta(\tau):=\frac{1}{\Gamma(\omega)} \int_{a}^{\tau}(\tau-s)^{\omega-1} \zeta(s) \Delta s
$$

where $\Gamma$ is the gamma function.

Definition 2.8 (Caputo fractional derivative on time scales) Let $\mathbb{T}$ be a time scale, $\tau \in \mathbb{T}$, $0<\omega<1$, and $\zeta: \mathbb{T} \rightarrow \mathbb{R}$. The Caputo $\Delta$ - fractional derivative of order $\omega$ of $\zeta$ is defined by

$$
{ }^{c} \Delta_{a^{+}}^{\omega} \zeta(\tau):=\frac{1}{\Gamma(n-\omega)} \int_{a}^{\tau}(\tau-s)^{n-\omega-1} \zeta^{\Delta^{n}}(s) \Delta s,
$$

where $n=[\omega]+1$ and $[\omega]$ denotes the integer part of $\omega$.

Theorem 2.9 (Semigroup property) Let $\omega, \varpi>0$ and $\zeta$ be an integrable function on $[a, b]$. Then

$$
{ }^{\mathbb{T}} I_{a}^{\omega \mathbb{T}} I_{a}^{\varpi} \zeta(\tau)={ }^{\mathbb{T}} I_{a}^{\omega+\infty} \zeta(\tau)
$$

Lemma 2.10 (Nonlinear alternative of Leray-Schauder type $[1,4,5])$ Let $E$ be a Banach space, $C$ be a closed, convex subset of $E, U$ be an open subset of $C$, and $0 \in U$. Suppose that $F: \bar{U} \rightarrow C$ is a continuous, compact map (that is, the image of any bounded subset is relatively compact). Then either 
(i) F has a fixed point in $\bar{U}$, or

(ii) there is $u \in \partial U$ (the boundary of $U$ in $C$ ) and $\lambda \in(0,1)$ with $u=\lambda F(u)$.

Lemma 2.11 (Krasnoselskii's fixed point theorem $[1,4,5]$ ) Let $S$ be a closed, bounded, convex, and nonempty subset of a Banach space X. Let $A, B$ be the operators such that (a) $A x+B x \in S$ whenever $x, y \in S ;(b) A$ is compact and continuous; $(c) B$ is a contraction mapping. Then there exists $z \in S$ such that $z=A z+B z$.

\section{Existence results}

Let $\widetilde{E}=\left\{u: u \in \mathcal{C}\left([-\epsilon, T]_{\mathbb{T}}, \mathbb{R}\right),{ }^{c} \Delta^{\omega}(u) \in \mathcal{C}\left([0, T]_{\mathbb{T}}, \mathbb{R}\right),{ }^{c} \Delta^{\omega}[u(\cdot)-\phi(\cdot, u)] \in \mathcal{C}\left([0, T]_{\mathbb{T}}, \mathbb{R}\right)\right\}$.

Definition 3.1 A function $u \in \widetilde{E}$ is said to be a solution of (1)-(3) if $u$ satisfies the equation ${ }^{c} \Delta^{\omega}\left[{ }^{c} \Delta^{\sigma} u(\vartheta)-\Phi\left(\vartheta, u_{\vartheta}\right)\right]=\Psi\left(\vartheta, u_{\vartheta}\right)$ on $\mathfrak{J}$, the condition $u(\vartheta)=\zeta(\vartheta)$ on $[-\epsilon, 0]_{\mathbb{T}}$ and ${ }^{c} \Delta^{\varpi} u(0)=\phi$.

Lemma 3.2 The function $u \in \widetilde{E}$ is a solution of the problem

$$
\begin{aligned}
& { }^{c} \Delta^{\omega}\left[{ }^{c} \Delta^{\varpi} u(\vartheta)-\Phi\left(\vartheta, u_{\vartheta}\right)\right]=\Psi\left(\vartheta, u_{\vartheta}\right), \quad \vartheta \in \mathfrak{J}, \\
& u(\vartheta)=\zeta(\vartheta), \quad \vartheta \in[-\epsilon, 0]_{\mathbb{T}}, \\
& { }^{c} \Delta^{\varpi} u(0)=\phi \in \mathbb{R},
\end{aligned}
$$

if and only if

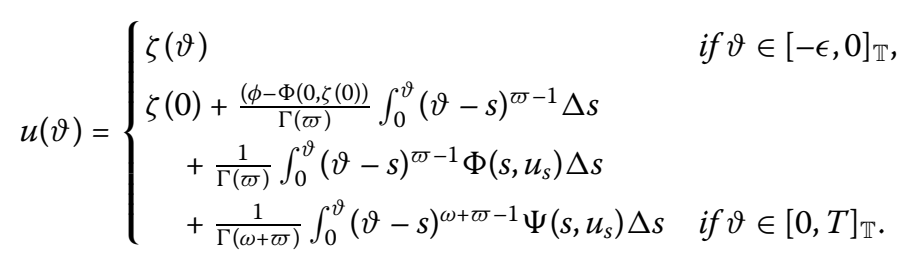

Proof Using ${ }^{c} \Delta^{\omega}\left[{ }^{c} \Delta^{\varpi} u(\vartheta)-\Phi\left(\vartheta, u_{\vartheta}\right)\right]=\Psi\left(\vartheta, u_{\vartheta}\right), \vartheta \in \mathfrak{J}$, we get

$$
{ }^{c} \Delta^{\varpi} u(\vartheta)-\Phi\left(\vartheta, u_{\vartheta}\right)=\alpha+\frac{1}{\Gamma(\omega)} \int_{0}^{\vartheta}(\vartheta-s)^{\omega-1} \Psi\left(s, u_{s}\right) \Delta s,
$$

where $\alpha \in \mathbb{R}$. From the condition $D^{\sigma} u(0)=\phi$ we find that $\alpha=\phi-\Phi(0, \zeta(0))$. Then

$$
{ }^{c} \Delta^{\varpi} u(\vartheta)=\phi-\Phi(0, \zeta(0))+\Phi\left(\vartheta, u_{\vartheta}\right)+\frac{1}{\Gamma(\omega)} \int_{0}^{\vartheta}(\vartheta-s)^{\omega-1} \Psi\left(s, u_{s}\right) \Delta s .
$$

Thus

$$
\begin{aligned}
u(\vartheta)= & \beta+\frac{(\phi-\Phi(0, \zeta(0))}{\Gamma(\varpi)} \int_{0}^{\vartheta}(\vartheta-s)^{\varpi-1} \Delta s+\frac{1}{\Gamma(\varpi)} \int_{0}^{\vartheta}(\vartheta-s)^{\varpi-1} \Phi\left(s, u_{s}\right) \Delta s \\
& +\frac{1}{\Gamma(\omega+\varpi)} \int_{0}^{\vartheta}(\vartheta-s)^{\omega+\varpi-1} \Psi\left(s, u_{s}\right) \Delta s .
\end{aligned}
$$

We find $\beta=\zeta(0)$, and (6) is proved. The converse follows by direct computation.

Assumptions: We need the following assumptions: 
(Ax.1) There exists $\lambda>0$ such that

$$
|\Psi(\vartheta, x)-\Psi(\vartheta, \bar{x})| \leq \lambda\|x-\bar{x}\|_{C} \quad \text { for } \vartheta \in \mathfrak{J} \text { and every } x, \bar{x} \in C_{\epsilon}
$$

(Ax.2) There exists a nonnegative constant $\mu$ such that

$$
|\Phi(\vartheta, x)-\Phi(\vartheta, \bar{x})| \leq \mu\|x-\bar{x}\|_{C} \quad \text { for } \vartheta \in \mathfrak{J} \text { and every } x, \bar{x} \in C_{\epsilon}
$$

(Ax.3) $\Psi, \Phi: \mathfrak{J} \times C_{\epsilon} \rightarrow \mathbb{R}$ are continuous functions;

(Ax.4) There exist a continuous nondecreasing function $\psi:[0, \infty)_{\mathbb{T}} \rightarrow(0, \infty)$ and a function $\Upsilon \in C\left(\mathfrak{J}, \mathbb{R}^{+}\right)$such that

$$
|\Psi(\vartheta, x)| \leq \Upsilon(\vartheta) \psi\left(\|x\|_{C}\right) \quad \text { for each }(\vartheta, x) \in \mathfrak{J} \times C_{\epsilon} ;
$$

(Ax.5) There exists a constant $\mathbf{L}>0$ such that

$$
\frac{\left(\Gamma(\varpi+1)-\mu T^{\varpi}\right) \mathbf{L}}{\mathbf{H}+\psi(\mathbf{L}) T^{\omega+\varpi}\|\Upsilon\|_{\infty} \frac{\Gamma(\varpi+1)}{\Gamma(\omega+\varpi+1)}}>1
$$

where

$$
\mathbf{H}=\Gamma(\varpi+1)\|\zeta\|_{C}+\left[|\phi|+\mu\|\zeta\|_{C}+2 \Phi_{0}\right] T^{\varpi} .
$$

Remark 3.3 Вy $(A x .2)$, for each $(\vartheta, x) \in \mathfrak{J}$,

$$
\begin{aligned}
|\Phi(\vartheta, x)| & =|\Phi(\vartheta, x)-\Phi(\vartheta, 0)+\Phi(\vartheta, 0)| \\
& \leq|\Phi(\vartheta, x)-\Phi(\vartheta, 0)|+|\Phi(\vartheta, 0)| \\
& \leq \mu|x|+\Phi_{0},
\end{aligned}
$$

where $\Phi_{0}=\sup _{s \in[0, T]_{\mathbb{T}}}|\Phi(s, 0)|$.

We establish our existence results for IVP (1)-(3). The first result is based on the Banach contraction principle.

Theorem 3.4 Assume that (Ax.1)-(Ax.2) hold. If

$$
T^{\omega}\left(\frac{\mu}{\Gamma(\varpi+1)}+\frac{\lambda T^{\varpi}}{\Gamma(\omega+\varpi+1)}\right)<1,
$$

then there exists a unique solution for IVP (1)-(3) defined on $\widetilde{E}$.

Proof Define the operator $\Xi: C\left([-\epsilon, T]_{\mathbb{T}}, \mathbb{R}\right) \rightarrow C\left([-\epsilon, T]_{\mathbb{T}}, \mathbb{R}\right)$ by

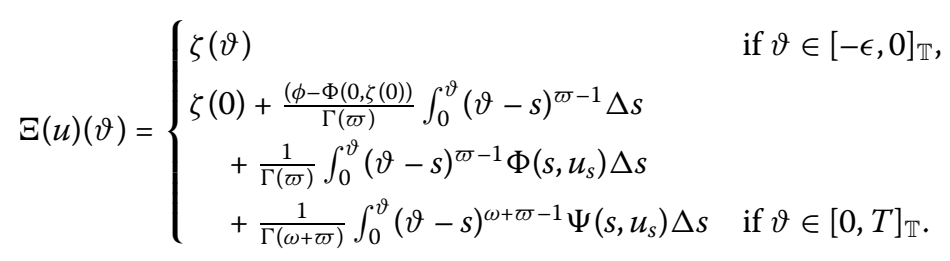


Let $u, v \in C\left([0, T]_{\mathbb{T}}, \mathbb{R}\right)$. Then, by $(A x .1),(A x .2)$, we get

$$
\begin{aligned}
|\Xi(u)(\vartheta)-\Xi(v)(\vartheta)| \leq & \frac{1}{\Gamma(\varpi)} \int_{0}^{\vartheta}(\vartheta-s)^{\varpi-1}\left|\Phi\left(s, u_{s}\right)-\Phi\left(s, v_{s}\right)\right| \Delta s \\
& +\frac{1}{\Gamma(\omega+\varpi)} \int_{0}^{\vartheta}(\vartheta-s)^{\omega+\varpi-1}\left|\Psi\left(s, u_{s}\right)-\Psi\left(s, v_{s}\right)\right| \Delta s \\
\leq & \frac{\mu}{\Gamma(\varpi)} \int_{0}^{\vartheta}(\vartheta-s)^{\varpi-1}\left\|u_{s}-v_{s}\right\|_{C} \Delta s \\
& +\frac{\lambda}{\Gamma(\omega+\varpi)} \int_{0}^{\vartheta}(\vartheta-s)^{\omega+\varpi-1}\left\|u_{s}-v_{s}\right\|_{C} \Delta s .
\end{aligned}
$$

On the other hand, by Lemma 2.6 we deduce

$$
\begin{aligned}
|\Xi(u)(\vartheta)-\Xi(v)(\vartheta)| \leq & \frac{\mu}{\Gamma(\varpi)} \int_{0}^{\vartheta}(\vartheta-s)^{\varpi-1}\left\|u_{s}-v_{s}\right\|_{C} d s \\
& +\frac{\lambda}{\Gamma(\omega+\varpi)} \int_{0}^{\vartheta}(\vartheta-s)^{\omega+\varpi-1}\left\|u_{s}-v_{s}\right\|_{C} d s \\
\leq & \frac{\mu \vartheta^{\varpi}}{\Gamma(\varpi+1)}\|u-v\|_{[-\epsilon, T]_{\mathbb{T}}}+\frac{\lambda \vartheta^{\omega+\varpi}}{\Gamma(\omega+\varpi+1)}\|u-v\|_{[0, T]_{\mathbb{T}}} \\
\leq & \frac{\mu T^{\varpi}}{\Gamma(\varpi+1)}\|u-v\|_{[-\epsilon, T]_{\mathbb{T}}}+\frac{\lambda T^{\omega+\varpi}}{\Gamma(\omega+\varpi+1)}\|u-v\|_{[0, T]_{\mathbb{T}}} .
\end{aligned}
$$

Thus

$$
\|\Xi(u)-\Xi(v)\|_{[-\epsilon, T]_{\mathbb{T}}} \leq T^{\omega}\left(\frac{\mu}{\Gamma(\varpi+1)}+\frac{\lambda T^{\varpi}}{\Gamma(\omega+\varpi+1)}\right)\|u-v\|_{[-\epsilon, T]_{\mathbb{T}}} .
$$

By (8), the operator $\Xi$ is a contraction. Hence, by Banach's contraction principle, $\Xi$ has a unique fixed point, which is a unique solution on $[-\epsilon, T]_{\mathbb{T}}$ of problem (1)-(3).

The second result is based on Leray-Schauder nonlinear alternative. Before the statement, we recall the notion of completely continuous. A bounded linear operator $T$ from Banach space $X$ to Banach space $Y$ is called completely continuous if, for every weakly convergent sequence $\left(x_{n}\right)$ from $X$, the sequence $\left(T x_{n}\right)$ is norm-convergent in $Y$.

Theorem 3.5 Assume that hypotheses (A2)-(A5) hold.If

$$
\frac{\mu T^{\varpi}}{\Gamma(\varpi+1)}<1
$$

then IVP (1)-(3) has at least one solution defined on $\widetilde{E}$.

Proof We shall show that the operator $\Xi: C\left([-\epsilon, T]_{\mathbb{T}}, \mathbb{R}\right) \rightarrow C\left([-\epsilon, T]_{\mathbb{T}}, \mathbb{R}\right)$ defined by (9) is continuous and completely continuous.

Claim 1: $\Xi$ is continuous. Let $\left\{u_{n}\right\}$ be a sequence such that $u_{n} \rightarrow y$ in $C\left([-\epsilon, T]_{\mathbb{T}}, \mathbb{R}\right)$. Then

$$
\left|\Xi\left(u_{n}\right)(\vartheta)-\Xi(u)(\vartheta)\right|
$$




$$
\begin{aligned}
\leq & \frac{1}{\Gamma(\varpi)} \int_{0}^{\vartheta}(\vartheta-s)^{\varpi-1}\left|\Phi\left(s, u_{n s}\right)-\Phi\left(s, u_{s}\right)\right| \Delta s \\
& +\frac{1}{\Gamma(\omega+\varpi)} \int_{0}^{\vartheta}(\vartheta-s)^{\omega+\varpi-1}\left|\Psi\left(s, u_{n s}\right)-\Psi\left(s, u_{s}\right)\right| \Delta s \\
\leq & \frac{1}{\Gamma(\varpi)} \int_{0}^{T}(\vartheta-s)^{\varpi-1} \sup _{s \in[0, T]_{\mathbb{T}}}\left|\Phi\left(s, u_{n s}\right)-\Phi\left(s, u_{s}\right)\right| \Delta s \\
& +\frac{1}{\Gamma(\omega+\varpi)} \int_{0}^{T}(\vartheta-s)^{\omega+\varpi-1} \sup _{s \in[0, T]_{\mathbb{T}}}\left|\Psi\left(s, u_{n s}\right)-\Psi\left(s, u_{s}\right)\right| \Delta s \\
\leq & \frac{\left\|\Phi\left(\cdot, u_{n .}\right)-\Phi(\cdot, u)\right\|_{\infty}}{\Gamma(\varpi)} \int_{0}^{T}(\vartheta-s)^{\omega-1} \Delta s \\
& +\frac{\left\|\Psi\left(\cdot, u_{n .}\right)-\Psi(\cdot, u .)\right\|_{\infty}}{\Gamma(\omega+\varpi)} \int_{0}^{T}(\vartheta-s)^{\omega+\varpi-1} \Delta s .
\end{aligned}
$$

And by Lemma 2.6 we deduce

$$
\begin{aligned}
\left|\Xi\left(u_{n}\right)(\vartheta)-\Xi(u)(\vartheta)\right| & \\
\leq & \frac{\left\|\Phi\left(\cdot, u_{n .}\right)-\Phi(\cdot, u .)\right\|_{\infty}}{\Gamma(\varpi)} \int_{0}^{T}(\vartheta-s)^{\omega-1} d s \\
& +\frac{\left\|\Psi\left(\cdot, u_{n .}\right)-\Psi(\cdot, u .)\right\|_{\infty}}{\Gamma(\omega+\varpi)} \int_{0}^{T}(\vartheta-s)^{\omega+\varpi-1} d s \\
\leq & \frac{T^{\varpi}\left\|\Phi\left(\cdot, u_{n .}\right)-\Phi\left(\cdot, u_{.}\right)\right\|_{\infty}}{\Gamma(\varpi+1)}+\frac{T^{\omega+\varpi}\left\|\Psi\left(\cdot, u_{n}\right)-\Psi(\cdot, u .)\right\|_{\infty}}{\Gamma(\omega+\varpi+1)} .
\end{aligned}
$$

Since $\Psi, \Phi$ are continuous functions, we have

$$
\begin{aligned}
& \left\|\Xi\left(u_{n}\right)-\Xi(u)\right\|_{\infty} \\
& \quad \leq \frac{T^{\varpi}\left\|\Phi\left(\cdot, u_{n}\right)-\Phi(\cdot, u .)\right\|_{\infty}}{\Gamma(\varpi+1)}+\frac{T^{\omega+\varpi}\left\|\Psi\left(\cdot, u_{n}\right)-\Psi(\cdot, u .)\right\|_{\infty}}{\Gamma(\omega+\varpi+1)} \rightarrow 0
\end{aligned}
$$

as $n \rightarrow \infty$.

Claim 2: $\Xi$ maps bounded sets into bounded sets in $C\left([-\epsilon, T]_{\mathbb{T}}, \mathbb{R}\right)$. Indeed, it is sufficient to show that for any $\kappa>0$ there exists a positive constant $\tilde{\lambda}$ such that, for each $u \in \mathcal{B}_{\kappa}=$ $\left\{u \in C\left([-\epsilon, T]_{\mathbb{T}}, \mathbb{R}\right):\|u\|_{\infty} \leq \kappa\right\}$, we have $\|\Xi(u)\|_{\infty} \leq \tilde{\lambda}$. By (A4) and (A5), for each $\vartheta \in \mathfrak{J}$, we have

$$
\begin{aligned}
|\Xi(u)(\vartheta)| \leq & \|\zeta\|_{C}+\frac{|\phi|+\mu\|\zeta\|_{C}+\Phi_{0}}{\Gamma(\varpi)} \int_{0}^{\vartheta}(\vartheta-s)^{\varpi-1} \Delta s \\
& +\frac{1}{\Gamma(\varpi)} \int_{0}^{\vartheta}(\vartheta-s)^{\varpi-1}\left|\Phi\left(s, u_{s}\right)\right| \Delta s \\
& +\frac{1}{\Gamma(\omega+\varpi)} \int_{0}^{\vartheta}(\vartheta-s)^{\omega+\varpi-1}\left|\Psi\left(s, u_{s}\right)\right| \Delta s \\
& \leq\|\zeta\|_{C}+\frac{|\phi|+\mu\|\zeta\|_{C}+\Phi_{0}}{\Gamma(\varpi)} \int_{0}^{\vartheta}(\vartheta-s)^{\varpi-1} \Delta s \\
& +\frac{\mu\|u\|_{[-\epsilon, T]_{\mathbb{T}}}+\Phi_{0}}{\Gamma(\varpi)} \int_{0}^{\vartheta}(\vartheta-s)^{\varpi-1} \Delta s
\end{aligned}
$$




$$
+\frac{\psi\left(\|u\|_{[-\epsilon, T]_{\mathbb{T}}}\right)\|\Upsilon\|_{\infty}}{\Gamma(\omega+\varpi)} \int_{0}^{\vartheta}(\vartheta-s)^{\omega+\varpi-1} \Delta s
$$

and by Lemma 2.6 we get

$$
\begin{aligned}
|\Xi(u)(\vartheta)| \leq & \|\zeta\|_{C}+\frac{|\phi|+\mu\|\zeta\|_{C}+\Phi_{0}}{\Gamma(\varpi)} \int_{0}^{\vartheta}(\vartheta-s)^{\varpi-1} d s \\
& +\frac{\mu\|u\|_{[-\epsilon, T]_{\mathbb{T}}}+\Phi_{0}}{\Gamma(\varpi)} \int_{0}^{\vartheta}(\vartheta-s)^{\varpi-1} d s \\
& +\frac{\psi\left(\|u\|_{\left.[-\epsilon, T]_{\mathbb{T}}\right)\|\Upsilon\|_{\infty}}^{\Gamma(\omega+\varpi)} \int_{0}^{\vartheta}(\vartheta-s)^{\omega+\varpi-1} d s\right.}{\leq} \\
& \|\zeta\|_{C}+\frac{\left[|\phi|+\mu\|\zeta\|_{C}+\Phi_{0}\right] T^{\varpi}}{\Gamma(\varpi+1)} \\
& +\frac{\left[\mu\|u\|_{[-\epsilon, T]_{T}}+\Phi_{0}\right] T^{\varpi}}{\Gamma(\varpi+1)}+\frac{\left[\psi\left(\|u\|_{[-\epsilon, T]_{\mathbb{T}}}\right)\|\Upsilon\|_{\infty}\right] T^{\omega+\varpi}}{\Gamma(\omega+\varpi+1)} .
\end{aligned}
$$

Thus

$$
\begin{aligned}
\|\Xi(u)\|_{\infty} \leq & \|\zeta\|_{C}+\frac{\left[|\phi|+\mu\|\zeta\|_{C}+\Phi_{0}\right] T^{\varpi}}{\Gamma(\varpi+1)} \\
& +\frac{\left(\mu \kappa+\Phi_{0}\right) T^{\omega}}{\Gamma(\varpi+1)}+\frac{\psi(\kappa)\|\Upsilon\|_{\infty} T^{\omega+\varpi}}{\Gamma(\omega+\varpi+1)}:=\tilde{\lambda} .
\end{aligned}
$$

Claim 3: $\Xi$ maps bounded sets into equicontinuous sets of $C\left([-\epsilon, T]_{\mathbb{T}}, \mathbb{R}\right)$. Let $\vartheta_{1}, \vartheta_{2} \in \mathfrak{J}$, $\vartheta_{1}<\vartheta_{2}, \mathcal{B}_{\kappa}$ be a bounded set of $C\left([-\epsilon, T]_{\mathbb{T}}, \mathbb{R}\right)$ as in Step 2 , and let $u \in \mathcal{B}_{\kappa}$. Then

$$
\begin{aligned}
\left|\Xi(u)\left(\vartheta_{2}\right)-\Xi(u)\left(\vartheta_{1}\right)\right| & \leq \frac{\mid(\phi-\Phi(0, \zeta(0)) \mid}{\Gamma(\varpi)} \int_{0}^{\vartheta_{1}}\left(\left(\vartheta_{2}-s\right)^{\varpi-1}-\left(\vartheta_{1}-s\right)^{\varpi-1}\right) \Delta s \\
& +\frac{\mid(\phi-\Phi(0, \zeta(0)) \mid}{\Gamma(\varpi)} \int_{\vartheta_{1}}^{\vartheta_{2}}\left(\vartheta_{2}-s\right)^{\varpi-1} \Delta s \\
& +\frac{1}{\Gamma(\varpi)} \int_{0}^{\vartheta_{1}}\left[\left(\vartheta_{2}-s\right)^{\varpi-1}-\left(\vartheta_{1}-s\right)^{\varpi-1}\right]\left|\Phi\left(s, u_{s}\right)\right| \Delta s \\
& +\frac{1}{\Gamma(\varpi)} \int_{\vartheta_{1}}^{\vartheta_{2}}\left(\vartheta_{2}-s\right)^{\omega-1}\left|\Phi\left(s, u_{s}\right)\right| \Delta s \\
& +\frac{1}{\Gamma(\omega+\varpi)} \int_{0}^{\vartheta_{1}}\left[\left(\vartheta_{2}-s\right)^{\omega+\varpi-1}-\left(\vartheta_{1}-s\right)^{\omega+\varpi-1}\right]\left|\Psi\left(s, u_{s}\right)\right| \Delta s \\
& +\frac{1}{\Gamma(\omega+\varpi)} \int_{\vartheta_{1}}^{\vartheta_{2}}\left(\vartheta_{2}-s\right)^{\omega+\varpi-1}\left|\Psi\left(s, u_{s}\right)\right| \Delta s,
\end{aligned}
$$

and by Lemma 2.6, we get

$$
\begin{aligned}
& \left|\Xi(u)\left(\vartheta_{2}\right)-\Xi(u)\left(\vartheta_{1}\right)\right| \\
& \leq \frac{\mid(\phi-\Phi(0, \zeta(0)) \mid}{\Gamma(\varpi)} \int_{0}^{\vartheta_{1}}\left(\left(\vartheta_{2}-s\right)^{\varpi-1}-\left(\vartheta_{1}-s\right)^{\varpi-1}\right) d s \\
& \quad+\frac{\mid(\phi-\Phi(0, \zeta(0)) \mid}{\Gamma(\varpi)} \int_{\vartheta_{1}}^{\vartheta_{2}}\left(\vartheta_{2}-s\right)^{\varpi-1} d s
\end{aligned}
$$




$$
\begin{aligned}
& +\frac{1}{\Gamma(\varpi)} \int_{0}^{\vartheta_{1}}\left[\left(\vartheta_{2}-s\right)^{\varpi-1}-\left(\vartheta_{1}-s\right)^{\varpi-1}\right]\left|\Phi\left(s, u_{s}\right)\right| d s \\
& +\frac{1}{\Gamma(\varpi)} \int_{\vartheta_{1}}^{\vartheta_{2}}\left(\vartheta_{2}-s\right)^{\omega-1}\left|\Phi\left(s, u_{s}\right)\right| d s \\
& +\frac{1}{\Gamma(\omega+\varpi)} \int_{0}^{\vartheta_{1}}\left[\left(\vartheta_{2}-s\right)^{\omega+\varpi-1}-\left(\vartheta_{1}-s\right)^{\omega+\varpi-1}\right]\left|\Psi\left(s, u_{s}\right)\right| d s \\
& +\frac{1}{\Gamma(\omega+\varpi)} \int_{\vartheta_{1}}^{\vartheta_{2}}\left(\vartheta_{2}-s\right)^{\omega+\varpi-1}\left|\Psi\left(s, u_{s}\right)\right| d s .
\end{aligned}
$$

Thus

$$
\begin{aligned}
& \left|\Xi(u)\left(\vartheta_{2}\right)-\Xi(u)\left(\vartheta_{1}\right)\right| \\
& \quad \leq \frac{|\phi|+\mu\|\zeta\|_{C}+\Phi_{0}}{\Gamma(\varpi+1)}\left[\vartheta_{2}^{\varpi}-\vartheta_{1}^{\varpi}\right] \\
& \quad+\frac{\mu \kappa+\Phi_{0}}{\Gamma(\varpi+1)}\left[\left|\vartheta_{2}^{\varpi}-\vartheta_{1}^{\varpi}\right|+\left|\vartheta_{2}-\vartheta_{1}\right|^{\varpi}\right] \\
& \quad+\frac{\psi(\kappa)\|\Upsilon\|_{\infty}}{\Gamma(\omega+\varpi+1)}\left[\left|\vartheta_{2}^{\omega+\varpi}-\vartheta_{1}^{\omega+\varpi}\right|+\left|\vartheta_{2}-\vartheta_{1}\right|^{\omega+\varpi}\right] .
\end{aligned}
$$

As $\vartheta_{1} \rightarrow \vartheta_{2}$, the right-hand side of the above inequality tends to zero. The equicontinuity for the cases $\vartheta_{1}<\vartheta_{2} \leq 0$ and $\vartheta_{1} \leq 0 \leq \vartheta_{2}$ is obvious. As a consequence of Steps 1 to 3 , it follows by the Arzelá-Ascoli theorem that $\Xi: C\left([-\epsilon, T]_{\mathbb{T}}, \mathbb{R}\right) \rightarrow C\left([-\epsilon, T]_{\mathbb{T}}, \mathbb{R}\right)$ is continuous and completely continuous.

Claim 4: We show that there exists an open set $U \subseteq C\left([-\epsilon, T]_{\mathbb{T}}, \mathbb{R}\right)$ with $u \neq \ell \Xi(u)$ for $\ell \in(0,1)$ and $u \in \partial U$. Let $u \in C\left([-\epsilon, T]_{\mathbb{T}}, \mathbb{R}\right)$ and $u=\ell \Xi(u)$ for some $0<\ell<1$. Then, for each $\vartheta \in \mathfrak{J}$, we have

$$
\begin{aligned}
u(\vartheta)= & \ell\left(\zeta(0)+(\phi-\Phi(0, \zeta(0))) \frac{\int_{0}^{\vartheta}(\vartheta-s)^{\varpi-1} \Delta s}{\Gamma(\varpi)}+\frac{1}{\Gamma(\varpi)} \int_{0}^{\vartheta}(\vartheta-s)^{\varpi-1} \Phi\left(s, u_{s}\right) \Delta s\right. \\
& \left.+\frac{1}{\Gamma(\omega+\varpi)} \int_{0}^{\vartheta}(\vartheta-s)^{\omega+\varpi-1} \Psi\left(s, u_{s}\right) \Delta s\right) .
\end{aligned}
$$

By our assumptions, for each $\vartheta \in \mathfrak{J}$, we obtain

$$
\begin{aligned}
|u(\vartheta)| \leq & \|\zeta\|_{C}+\left[|\phi|+\mu\|\zeta\|_{C}+\Phi_{0}\right] \frac{\int_{0}^{\vartheta}(\vartheta-s)^{\varpi-1} \Delta s}{\Gamma(\varpi)} \\
& +\frac{\mu\|u\|_{[-\epsilon, T]_{\mathbb{T}}}+\Phi_{0}}{\Gamma(\varpi)} \int_{0}^{\vartheta}(\vartheta-s)^{\varpi-1} \Delta s \\
& +\frac{1}{\Gamma(\omega+\varpi)} \int_{0}^{\vartheta}(\vartheta-s)^{\omega+\varpi-1} \Upsilon(s) \psi\left(\left\|u_{s}\right\|_{C}\right) \Delta s \\
\leq & \|\zeta\|_{C}+\left[|\phi|+\mu\|\zeta\|_{C}+\Phi_{0}\right] \frac{\int_{0}^{\vartheta}(\vartheta-s)^{\varpi-1} d s}{\Gamma(\varpi)} \\
& +\frac{\mu\|u\|_{[-\epsilon, T]_{\mathbb{T}}}+\Phi_{0}}{\Gamma(\varpi)} \int_{0}^{\vartheta}(\vartheta-s)^{\varpi-1} d s \\
& +\frac{1}{\Gamma(\omega+\varpi)} \int_{0}^{\vartheta}(\vartheta-s)^{\omega+\varpi-1} \Upsilon(s) \psi\left(\left\|u_{s}\right\|_{C}\right) d s
\end{aligned}
$$




$$
\begin{aligned}
\leq & \|\zeta\|_{C}+\frac{\left[|\phi|+\mu\|\zeta\|_{C}+\Phi_{0}\right] T^{\varpi}}{\Gamma(\varpi+1)} \\
& +\frac{\mu\|u\|_{[-\epsilon, T]_{\mathbb{T}}}+\Phi_{0}}{\Gamma(\varpi+1)} T^{\varpi}+\frac{\|\Upsilon\|_{\infty} \psi\left(\|u\|_{[-\epsilon, T]_{\mathbb{T}}}\right)}{\Gamma(\omega+\varpi+1)} T^{\omega+\varpi} .
\end{aligned}
$$

Then

$$
\begin{aligned}
\|u\|_{[-\epsilon, T]_{\mathbb{T}} \leq} & \|\zeta\|_{C}+\frac{\left[|\phi|+\mu\|\zeta\|_{C}+\Phi_{0}\right] T^{\varpi}}{\Gamma(\varpi+1)} \\
& +\frac{\mu\|u\|_{[-\epsilon, T]_{\mathbb{T}}}+\Phi_{0}}{\Gamma(\varpi+1)} T^{\varpi}+\frac{\|\Upsilon\|_{\infty} \psi\left(\|u\|_{[-\epsilon, T]_{\mathbb{T}}}\right)}{\Gamma(\omega+\varpi+1)} T^{\omega+\varpi} .
\end{aligned}
$$

Thus

$$
\begin{aligned}
\left(\Gamma(\varpi+1)-\mu T^{\varpi}\right)\|u\|_{[-\epsilon, T]_{\mathbb{T}} \leq} & \Gamma(\varpi+1)\|\zeta\|_{C}+\left[|\phi|+\mu\|\zeta\|_{C}+2 \Phi_{0}\right] T^{\varpi} \\
& +T^{\omega+\varpi}\|\Upsilon\|_{\infty} \psi\left(\|y\|_{[-\epsilon, T]_{\mathbb{T}}}\right) \frac{\Gamma(\varpi+1)}{\Gamma(\omega+\varpi+1)}
\end{aligned}
$$

which can be expressed as

$$
\frac{\left(\Gamma(\varpi+1)-\mu T^{\varpi}\right)\|u\|_{[-\epsilon, T]_{\mathbb{T}}}}{\mathbf{H}+T^{\omega+\varpi} \psi\left(\|u\|_{[-\epsilon, T]_{\mathbb{T}}}\right)\|\Upsilon\|_{\infty} \frac{\Gamma(\varpi+1)}{\Gamma(\omega+\varpi+1)}} \leq 1 .
$$

In view of (A6), there exists $\mathbf{L}$ such that $\|u\|_{[-\epsilon, T]_{\mathbb{T}}} \neq \mathbf{L}$. Let us set

$$
U=\left\{u \in C\left([-\epsilon, T]_{\mathbb{T}}, \mathbb{R}\right):\|u\|_{[-\epsilon, T]_{\mathbb{T}}}<\mathbf{L}\right\} .
$$

Moreover the operator $\Xi: \bar{U} \rightarrow C\left([-\epsilon, T]_{\mathbb{T}}, \mathbb{R}\right)$ is continuous and completely continuous. From the choice of $U$, there is no $u \in \partial U$ such that $u=\ell \Xi u$ for some $\ell \in(0,1)$. Thus, by the nonlinear alternative of Leray-Schauder type Lemma 2.10, we deduce that $\Xi$ has a fixed point $u \in \bar{U}$ which is a solution of problem (1)-(3).

The second existence result is based on Krasnoselskii's fixed point theorem.

Theorem 3.6 Assume that (Ax.2)-(Ax.3), (10) hold, and

(Ax.6) $|\Psi(\vartheta, x)| \leq \chi_{1}(\vartheta),|\Phi(\vartheta, x)| \leq \chi_{2}(\vartheta)$ for all $(\vartheta, x) \in \mathfrak{J} \times \mathbb{R}$, where

$$
\chi_{1}, \chi_{2} \in C\left(\mathfrak{J}, \mathbb{R}^{+}\right) .
$$

Then problem (1)-(3) has at least one solution defined on $\widetilde{E}$.

Proof Let the operators $\mathcal{Q}_{1}$ and $\mathcal{Q}_{2}$ :

$$
\begin{aligned}
& \mathcal{Q}_{1} u(\vartheta)=\left\{\begin{array}{ll}
0 & \text { if } \vartheta \in[-\epsilon, 0]_{\mathbb{T}}, \\
\left(\phi-\Phi(0, \zeta(0)) \frac{1}{\Gamma(\varpi)} \int_{0}^{\vartheta}(\vartheta-s)^{\varpi-1} \Delta s\right. & \text { if } \vartheta \in[0, T]_{\mathbb{T}},
\end{array},\right. \\
& \mathcal{Q}_{2} u(t)= \begin{cases}\zeta(\vartheta) & \text { if } \vartheta \in[-\epsilon, 0]_{\mathbb{T}}, \\
\zeta(0)+\frac{1}{\Gamma(\omega+\varpi)} \int_{0}^{\vartheta}(\vartheta-s)^{\varpi-1} \Phi\left(s, u_{s}\right) \Delta s & \text { if } \vartheta \in[0, T]_{\mathbb{T}} .\end{cases}
\end{aligned}
$$


Put

$$
\sup _{\vartheta \in[0, T]_{\mathbb{T}}} \chi_{1}(\vartheta)=\left\|\chi_{1}\right\|_{\infty}, \quad \sup _{\vartheta \in[0, T]_{\mathbb{T}}} \chi_{2}(\vartheta)=\left\|\chi_{2}\right\|_{\infty}
$$

and

$$
\varrho \geq\|\zeta\|_{C}+T^{\varpi}\left[\frac{\left[|\phi|+2\left\|\chi_{2}\right\|_{\infty}\right]}{\Gamma(\varpi+1)}+\frac{T^{\omega}\left\|\chi_{1}\right\|_{\infty}}{\Gamma(\omega+\varpi+1)}\right],
$$

and define $\mathcal{D}_{\varrho}=\left\{u \in C\left([\epsilon, T]_{\mathbb{T}}, \mathbb{R}\right):\|u\|_{\infty} \leq \varrho\right\}$.

Claim 1: For any $u, v \in \mathcal{D}_{\varrho}: \mathcal{Q}_{1} u+\mathcal{Q}_{2} v \in \mathcal{D}_{\varrho}$ : For any $u, v \in \mathcal{D}_{\varrho}$, by (11), (12), and Lemma 2.6, we have

$$
\begin{aligned}
\left|\mathcal{Q}_{1} u(\vartheta)+\mathcal{Q}_{2} v(\vartheta)\right| & \\
\leq & \sup _{\vartheta \in[0, T]_{\mathbb{T}}}\left\{\frac{(\phi-\Phi(0, \zeta(0))}{\Gamma(\varpi)} \int_{0}^{\vartheta}(\vartheta-s)^{\varpi-1} \Delta s+\frac{1}{\Gamma(\varpi)} \int_{0}^{\vartheta}(\vartheta-s)^{\omega-1} \Phi\left(s, u_{s}\right) \Delta s\right. \\
& \left.+\zeta(0)+\frac{1}{\Gamma(\omega+\varpi)} \int_{0}^{\vartheta}(\vartheta-s)^{\omega+\varpi-1} \Psi\left(s, v_{s}\right) \Delta s\right\} \\
\leq & \sup _{\vartheta \in[0, T]_{\mathbb{T}}}\left\{\frac{(\phi-\Phi(0, \zeta(0))}{\Gamma(\varpi)} \int_{0}^{\vartheta}(\vartheta-s)^{\varpi-1} d s+\frac{1}{\Gamma(\varpi)} \int_{0}^{\vartheta}(\vartheta-s)^{\omega-1} \Phi\left(s, u_{s}\right) d s\right. \\
& \left.+\zeta(0)+\frac{1}{\Gamma(\omega+\varpi)} \int_{0}^{\vartheta}(\vartheta-s)^{\omega+\varpi-1} \Psi\left(s, v_{s}\right) d s\right\} \\
\leq & \|\zeta\|_{C}+T^{\varpi}\left[\frac{\left[|\phi|+2\left\|\chi_{2}\right\|_{\infty}\right]}{\Gamma(\varpi+1)}+\frac{T^{\omega}\left\|\chi_{1}\right\|_{\infty}}{\Gamma(\omega+\varpi+1)}\right] \\
\leq & \varrho .
\end{aligned}
$$

This shows that $\mathcal{Q}_{1} y+\mathcal{Q}_{2} z \in \mathcal{D}_{\varrho}$.

Claim 2: $\mathcal{Q}_{1}$ is a contraction mapping on $\mathcal{D}_{\varrho}$ :

Let $u, v \in \mathcal{D}_{\varrho}$. Then, by (11) and Lemma 2.6,

$$
\begin{aligned}
\left|\mathcal{Q}_{1}(u)(\vartheta)-\mathcal{Q}_{1}(v)(\vartheta)\right| & \leq \frac{1}{\Gamma(\varpi)} \int_{0}^{\vartheta}(\vartheta-s)^{\varpi-1}\left|\Phi\left(s, u_{s}\right)-\Phi\left(s, v_{s}\right)\right| \Delta s \\
& \leq \frac{\mu}{\Gamma(\varpi)} \int_{0}^{\vartheta}(\vartheta-s)^{\varpi-1}\left\|u_{s}-v_{s}\right\|_{C} \Delta s \\
& \leq \frac{\mu}{\Gamma(\varpi)} \int_{0}^{\vartheta}(\vartheta-s)^{\varpi-1}\left\|u_{s}-v_{s}\right\|_{C} d s \\
& \leq \frac{\mu \vartheta^{\varpi}}{\Gamma(\varpi+1)}\|u-v\|_{[-\epsilon, T]_{\mathbb{T}}} \\
& \leq \frac{\mu T^{\varpi}}{\Gamma(\varpi+1)}\|u-v\|_{[-\epsilon, T]_{\mathbb{T}}} .
\end{aligned}
$$

Thus

$$
\left\|\mathcal{Q}_{1}(u)-\mathcal{Q}_{1}(v)\right\|_{[-\epsilon, T]_{\mathbb{T}}} \leq \frac{\mu T^{\omega}}{\Gamma(\varpi+1)}\|u-v\|_{[-\epsilon, T]_{\mathbb{T}}} .
$$

And by (10), $\mathcal{Q}_{1}$ is a contraction mapping. 
Claim 3: $\mathcal{Q}_{2}$ is continuous: Clearly $\mathcal{Q}_{2}$ is continuous because $\Psi$ is continuous. Moreover, $\mathcal{Q}_{2}$ is uniformly bounded on $D_{\varrho}$ as

$$
\left\|\mathcal{Q}_{2} u\right\| \leq\|\zeta\|_{C}+\frac{T^{\omega+\varpi}\left\|\chi_{1}\right\|_{\infty}}{\Gamma(\omega+\varpi+1)}
$$

Claim 4: $\mathcal{Q}_{2}$ is equicontinuous: Define

$$
\Psi_{0}=\sup _{(\vartheta, u) \in[0, T]_{\mathbb{T}} \times \mathcal{D}_{\varrho}}|\Psi(\vartheta, u)|<\infty .
$$

For $\vartheta_{1}, \vartheta_{2} \in[0, T]_{\mathbb{T}}, \vartheta_{1}<\vartheta_{2}$, by (12) and Lemma 2.6, we have

$$
\begin{aligned}
\left|\mathcal{Q}_{2} u\left(\vartheta_{2}\right)-\mathcal{Q}_{2} u\left(\vartheta_{1}\right)\right| & \\
\leq & \frac{\Psi_{0}}{\Gamma(\omega+\varpi)} \int_{0}^{\vartheta_{1}}\left|\left(\vartheta_{2}-s\right)^{\omega+\omega-1}-\left(\vartheta_{1}-s\right)^{\omega+\varpi-1}\right| \Delta s \\
& +\frac{\Psi_{0}}{\Gamma(\omega+\varpi)} \int_{\vartheta_{1}}^{\vartheta_{2}}\left(\vartheta_{2}-s\right)^{\omega+\varpi-1} \Delta s \\
\leq & \frac{\Psi_{0}}{\Gamma(\omega+\varpi)} \int_{0}^{\vartheta_{1}}\left|\left(\vartheta_{2}-s\right)^{\omega+\varpi-1}-\left(\vartheta_{1}-s\right)^{\omega+\varpi-1}\right| d s \\
& +\frac{\Psi_{0}}{\Gamma(\omega+\varpi)} \int_{\vartheta_{1}}^{\vartheta_{2}}\left(\vartheta_{2}-s\right)^{\omega+\varpi-1} d s \\
\leq & \frac{\Psi_{0}}{\Gamma(\omega+\varpi+1)}\left[\left|\vartheta_{2}^{\omega+\infty}-\vartheta_{1}^{\omega+\varpi}\right|+\left|\vartheta_{2}-\vartheta_{1}\right|^{\omega+\varpi}\right] .
\end{aligned}
$$

As $\vartheta_{1} \rightarrow \vartheta_{2}$, the right-hand side of the above inequality tends to zero. Thus, $\mathcal{Q}_{2}$ is equicontinuous. So $\mathcal{Q}_{2}$ is relatively compact on $\mathcal{D}_{\varrho}$. Hence, by the Arzelá-Ascoli theorem, $\mathcal{Q}_{2}$ is compact on $\mathcal{D}_{\varrho}$. So, by Lemma 2.11, problem (1)-(3) has at least one solution on $[-\epsilon, T]_{\mathbb{T}}$.

\section{Examples}

Example 1 Let $\mathbb{T}$ be a time scale and let us consider the fractional functional differential equation

$$
\begin{aligned}
& { }^{c} \Delta^{1 / 3}\left[{ }^{c} \Delta^{1 / 2} u(\vartheta)-\frac{1}{1000}\left(\vartheta \cos \left\|u_{\vartheta}\right\|_{C}-\left\|u_{\vartheta}\right\|_{C} \sin \vartheta\right)\right]=\frac{1}{100 e^{\vartheta}} \frac{\left\|u_{\vartheta}\right\|_{C}}{5+\left\|u_{\vartheta}\right\|_{C}}, \\
& \vartheta \in \mathfrak{J}:=[0,1] \cap \mathbb{T}, \\
& u(\vartheta)=\zeta(\vartheta), \quad \vartheta \in[-\epsilon, 0] \cap \mathbb{T}, \\
& D^{1 / 2} u(0)=1 / 3 .
\end{aligned}
$$

Let

$$
\begin{aligned}
& \Psi(\vartheta, x)=\frac{1}{100 e^{\vartheta}} \frac{x}{5+x}, \\
& \Phi(\vartheta, x)=\frac{1}{1000}(\vartheta \cos x-x \sin \vartheta), \quad(\vartheta, x) \in[0,1] \cap \mathbb{T} \times[0, \infty) .
\end{aligned}
$$


For $u, v \in[0, \infty)$ and $\vartheta \in \mathfrak{J}$, we have

$$
\begin{aligned}
|\Psi(\vartheta, u)-\Psi(\vartheta, v)| & =\frac{1}{100 e^{\vartheta}}\left|\frac{u}{5+u}-\frac{v}{5+v}\right| \\
& =\frac{5|u-v|}{100 e^{\vartheta}(5+u)(5+v)} \\
& \leq \frac{1}{500}|u-v|,
\end{aligned}
$$

and

$$
\begin{aligned}
|\Phi(\vartheta, u)-\Phi(\vartheta, v)| & \leq \frac{1}{1000}|\vartheta||\cos u-\cos v|+\frac{1}{1000}|\sin \vartheta||u-v| \\
& \leq \frac{1}{1000}|u-v|+\frac{1}{1000}|u-v| \\
& \leq \frac{1}{500}(|u-v|)
\end{aligned}
$$

And

$$
\begin{aligned}
T^{\omega}\left(\frac{\mu}{\Gamma(\varpi+1)}+\frac{\lambda T^{\varpi}}{\Gamma(\omega+\varpi+1)}\right) & =\left(\frac{\frac{1}{500}}{\Gamma(1 / 2+1)}+\frac{\frac{1}{500}}{\Gamma(1 / 3+1 / 2+1)}\right) \\
& =\frac{1}{500}\left(\frac{1}{\Gamma(3 / 2)}+\frac{1}{\Gamma(11 / 6)}\right) \\
& \approx 0.00438511892015845 \\
& <1 .
\end{aligned}
$$

Hence conditions (A1) and (A2) hold with $\lambda=\mu=\frac{1}{500}$. By Theorem 3.4, problem (14)(16) has a unique solution on $[-\epsilon, 1]_{\mathbb{T}}$.

Example 2 Let $\mathbb{T}$ be a time scale and let us consider the fractional functional differential equation

$$
\begin{aligned}
& { }^{c} \Delta^{1 / 5}\left[{ }^{c} \Delta^{1 / 2} u(\vartheta)-\frac{1}{100 e^{\vartheta}\left(1+\left\|u_{\vartheta}\right\|_{C}\right)}\right]=\frac{e^{-\vartheta}}{111+e^{\vartheta}} \frac{\left\|u_{\vartheta}\right\|_{C}}{1+\left\|u_{\vartheta}\right\|_{C}}, \\
& \vartheta \in \mathfrak{J}:=[0,1] \cap \mathbb{T}, \\
& u(\vartheta)=\zeta(\vartheta), \quad \vartheta \in[-\epsilon, 0] \cap \mathbb{T}, \\
& D^{1 / 2} u(0)=1 / 3 .
\end{aligned}
$$

Let

$$
\Psi(\vartheta, x)=\frac{e^{-\vartheta}}{111+e^{\vartheta}} \frac{x}{1+x}, \quad \Phi(\vartheta, x)=\frac{1}{100 e^{\vartheta}(1+x)}, \quad(\vartheta, x) \in[0,1] \cap \mathbb{T} \times[0, \infty) .
$$

(Ax.2) is clearly satisfied. Indeed, for $u, v \in[0, \infty)$ and $\vartheta \in \mathfrak{J}$, we have

$$
|\Phi(\vartheta, u)-\Phi(\vartheta, v)|=\frac{1}{100 e^{\vartheta}}\left|\frac{1}{1+u}-\frac{1}{1+v}\right|
$$




$$
\begin{aligned}
& =\frac{|u-v|}{100 e^{\vartheta}(1+u)(1+v)} \\
& \leq \frac{1}{100}|u-v| .
\end{aligned}
$$

Clearly,

$$
\begin{aligned}
& |\Psi(\vartheta, x)| \leq \frac{e^{-\vartheta}}{111+e^{\vartheta}}=\chi_{1}(\vartheta), \\
& |\Phi(\vartheta, x)| \leq \frac{1}{100 e^{\vartheta}}=\chi_{2}(\vartheta),
\end{aligned}
$$

and

$$
\frac{\mu T^{\varpi}}{\Gamma(\varpi+1)}=\frac{1}{100 \Gamma(3 / 2)}=\frac{1}{50 \sqrt{\pi}} \approx 0.0112837916709551<1 .
$$

Clearly, the assumptions of Theorem 3.6 are satisfied. Consequently, by the conclusion of Theorem 3.6, there exists a solution of problem (14)-(16) on $[-\epsilon, 1]_{\mathbb{T}}$.

\section{Acknowledgements}

Authors thank their own institutes. We are also grateful to the referees for the careful reading of the paper and for their helpful remarks and suggestions.

Funding

We declare that funding is not applicable for our paper.

Availability of data and materials

The data and material used to support the findings of this study are included within the article.

\section{Declarations}

\section{Competing interests}

The authors declare that they have no competing interests.

\section{Authors' contributions}

All authors contributed equally and significantly in writing this article. All authors read and approved the final manuscript.

\section{Author details}

${ }^{1}$ Laboratory of Mathematics, Djillali Liabes University of Sidi Bel-Abbes, P.O. Box 89, Sidi Bel Abbes, 22000, Algeria.

${ }^{2}$ Division of Applied Mathematics, Thu Dau Mot University, Thu Dau Mot, Binh Duong Province, Vietnam. ${ }^{3}$ Department of Mathematics, Çankaya University, 06790, Etimesgut, Ankara, Turkey.

\section{Publisher's Note}

Springer Nature remains neutral with regard to jurisdictional claims in published maps and institutional affiliations.

Received: 11 August 2021 Accepted: 18 January 2022 Published online: 01 March 2022

\section{References}

1. Abbas, S., Benchohra, M., Graef, J.R., Henderson, J.: Implicit Fractional Differential and Integral Equations Existence and Stability. de Gruyter, Berlin (2018)

2. Abbas, S., Benchohra, M., Lazreg, J.E., Alsaedi, A., Zhou, Y.: Existence and Ulam stability for fractional differential equations of Hilfer-Hadamard type. Adv. Differ. Equ. 2017, Article ID 180 (2017)

3. Abbas, S., Benchohra, M., Lazreg, J.E., Zhou, Y.: A survey on Hadamard and Hilfer fractional differential equations: analysis and stability. Chaos Solitons Fractals 102, 47-71 (2017)

4. Abbas, S., Benchohra, M., N'Guérékata, G.M.: Topics in Fractional Differential Equations. Springer, New York (2012)

5. Abbas, S., Benchohra, M., N'Guérékata, G.M.: Advanced Fractional Differential and Integral Equations. Nova Science Publishers, New York (2014)

6. Abdeljawad, A., Agarwal, R.P., Karapinar, E., Kumari, P.S.: Solutions of the nonlinear integral equation and fractional differential equation using the technique of a fixed point with a numerical experiment in extended b-metric space. Symmetry 11, 686 (2019) 
7. Adiguzel, R.S., Aksoy, U., Karapinar, E., Erhan, M.: On the solutions of fractional differential equations via Geraghty type hybrid contractions. Appl. Comput. Math. 20(2), 313-333 (2021)

8. Adiguzel, R.S., Aksoy, U., Karapinar, E., Erhan, M.: On the solution of a boundary value problem associated with a fractional differential equation. Math. Methods Appl. Sci. https://doi.org/10.1002/mma.665

9. Afshari, H., Kalantari, S., Karapinar, E.: Solution of fractional differential equations via coupled fixed point. Electron. J. Differ. Equ. 2015, Article ID 286 (2015)

10. Afshari, H., Karapınar, E.: A discussion on the existence of positive solutions of the boundary value problems via $\psi$-Hilfer fractional derivative on b-metric spaces. Adv. Differ. Equ. 2020, Article ID 616 (2020)

11. Agarwal, R., O'Regan, D., Saker, S.: Dynamic Inequalities on Time Scales. Springer, Berlin (2014)

12. Agarwal, R.P., Aksoy, U., Karapinar, E., Erhan, I.M.: F-contraction mappings on metric-like spaces in connection with integral equations on time scales. Rev. R. Acad. Cienc. Exactas Fís. Nat., Ser. A Mat. 114(3), Article ID 147 (2020)

13. Ahmad, B., Ntouyas, S.: Existence and uniqueness of solutions for Caputo-Hadamard sequential fractional order neutral functional differential equations. Electron. J. Differ. Equ. 2017, Article ID 36 (2017)

14. Ahmadkhanlu, A., Jahanshahi, M.: On the existence and uniqueness of solution of initial value problem for fractional order differential equations on time scales. Bull. Iranian Math. Soc. 38(1), 241-252 (2012)

15. Alqahtani, B., Aydi, H., Karapınar, E., Rakocevic, V.: A solution for Volterra fractional integral equations by hybrid contractions. Mathematics 7, 694 (2019)

16. Aulbach, B., Hilger, S.: A unified approach to continuous and discrete dynamics. In: Qualitative Theory of Differential Equations, Szeged, 1988. Colloq. Math. Soc. János Bolyai, vol. 53, pp. 37-56. North-Holland, Amsterdam (1990)

17. Baleanu, D., Güvenç, Z.B., Machado, J.A.T.: New Trends in Nanotechnology and Fractional Calculus Applications. Springer, New York (2010)

18. Benkhettou, N., Brito da Cruz, A.M.C., Torres, D.F.M.: A fractional calculus on arbitrary time scales: fractional differentiation and fractional integration. Signal Process. 107, 230-237 (2015)

19. Benkhettou, N., Hassani, S., Torres, D.F.M.: A conformable fractional calculus on arbitrary time scales. J. King Saud Univ. Sci. 28, 93-98 (2016)

20. Bohner, M., Georgiev, S.G.: Multivariable Dynamic Calculus on Time Scales. Springer, Berlin (2016)

21. Bohner, M., Peterson, A.: Dynamic Equations on Time Scales. Birkhäuser, Boston (2001)

22. Bohner, M., Peterson, A.: Advances in Dynamic Equations on Time Scales. Birkhäuser, Boston (2003)

23. Devaraj, V., Kuppusamy, K., Seenith, S.: On the behavior of solutions of fractional differential equations on time scale via Hilfer fractional derivatives. Fract. Calc. Appl. Anal. 21(4), 1120-1138 (2018)

24. Georgiev, S.: Fractional Dynamic Calculus and Fractional Dynamic Equations on Time Scales. Springer, Berlin (2018)

25. Georgiev, S.G.: Integral Equations on Time Scales. Atlantis Press, Paris (2016)

26. Georgiev, S.G.: Fractional Dynamic Calculus and Fractional Dynamic Equations on Time Scales. Springer, Berlin (2018)

27. Georgiev, S.G.: Functional Dynamic Equations on Time Scales. Springer, Berlin (2019)

28. Hilger, S.: Analysis on measure chains a unified approach to continuous and discrete calculus. Results Math. 18(1-2), 18-56 (1990)

29. Karapinar, E., Abdeljawad, T., Jarad, F.: Applying new fixed point theorems on fractional and ordinary differential equations. Adv. Differ. Equ. 2019, 421 (2019)

30. Karapinar, E., Duy Binh, H., Hoang Luc, N., Huu, N.: Can on continuity of the fractional derivative of the time-fractional semilinear pseudo-parabolic systems. Adv. Differ. Equ. 2021, 70 (2021). https://doi.org/10.1186/s13662-021-03232-z

31. Karapinar, E., Fulga, A., Rashid, M., Shahid, L., Aydi, H.: Large contractions on quasi-metric spaces with an application to nonlinear fractional differential equations. Mathematics 7, 444 (2019)

32. Vipin, K., Muslim, M.: Existence and stability results of nonlinear fractional differential equations with nonlinear integral boundary condition on time scales. Appl. Appl. Math. 2020(6), 129-145 (2020)

33. Wang, C., Agarwal, R.P., O'Regan, D., Sakthivel, R.: Theory of Translation Closedness for Time Scales. Springer, Berlin (2020)

34. Zhou, Y:: Basic Theory of Fractional Differential Equations. World Scientific, Singapore (2014)

\section{Submit your manuscript to a SpringerOpen ${ }^{\circ}$ journal and benefit from:}

- Convenient online submission

- Rigorous peer review

- Open access: articles freely available online

- High visibility within the field

- Retaining the copyright to your article

Submit your next manuscript at $\gg$ springeropen.com 